\title{
OXIDATION RESISTANT CERAMIC COMPOSITES: HOW WEAK INTERFACES BECAME DESIGNED FAILURE PROCESSES
}

\author{
RONALD J. KERANS \\ Air Force Research Laboratory, Materials and Manufacturing Directorate, AFRL/MLLN, \\ Wright-Patterson AFB OH 45433
}

\begin{abstract}
Interest in ceramic composites arises from their potential for dramatically improved damage tolerance, as compared to monolithic ceramics, while delivering similar high-temperature properties. The remarkable toughening effect resulting from the proper combination of brittle phases is dependent upon the failure process developing in a particular way; matrix cracks must deflect into fiber debonding cracks that run in or near the fiber/matrix interface and permit some relative displacement. In early composites the requirements for crack deflection and good composite behavior seem to have been almost fortuitously satisfied by native $\mathrm{C}$ layers; however, the most productive uses of ceramic composites are dependent upon the development of fiber coatings that not only promote the desired interfacial failure, but also satisfy the additional requirement of being oxidation resistant. Though conceptually simple, the management of a failure process-specifically, predetermining the progress of cracks on a very local level-places a very demanding and unprecedented requirement on the materials designer. Historically, the details of fracture events were of interest principally for insight into means of avoiding them. The composite problem imposes the new requirement that we design to promote a special kind of fracture, and control post-fracture fiction within a certain range. This truly is a higher level of sophistication than heretofore attained, and its complexities have deterred many investigators from seriously addressing the problem. Nevertheless, there has been progress both in depth of understanding and in actual achievement of viable systems. Moreover, very interesting properties of several families of materials have been uncovered. These issues will be outlined and discussed in more detail with the objective of developing a broad view of the lessons learned and opportunities revealed.
\end{abstract}

